\title{
Stability analysis of a giving up smoking model
}

\author{
Zainab Alkhudhari, Sarah Al-Sheikh*, Salma Al-Tuwairqi \\ Department of Mathematics, King Abdulaziz Universtiy, Jeddah 21551, Saudi Arabia \\ ${ }^{*}$ Corresponding autor E-mail: salsheikh@kau.edu.sa
}

Copyright (C) 2014 Zainab Alkhudhar et. al. This is an open access article distributed under the Creative Commons Attribution License, which permits unrestricted use, distribution, and reproduction in any medium, provided the original work is properly cited.

\begin{abstract}
In this paper we present and analyze a generalization of the giving up smoking model that was introduced by Sharomi and Gumel [4], in which quitting smoking can be temporary or permanent. In our model, we study a population with peer pressure effect on temporary quitters and we consider also the possibility of temporary quitters becoming permanent quitters and the impact of this transformation on the existence and stability of equilibrium points. Numerical results are given to support the results.
\end{abstract}

Keywords: Smoking model, Smoking generation number, Local stability, Global stability, Second additive compound matrix, Liapunov function.

\section{Introduction}

The World Health Organization estimates that tobacco causes approximately 5 million deaths annually worldwide, and this number is expected to double by 2025. The reason for that high number is that Tobacco use is a major cause of many of the world's top killer diseases including cardiovascular disease, chronic lung disease and lung cancer. Smoking is often the hidden cause of many killing diseases. In Saudi Arabia, the prevalence of current smoking ranges from 2.4-52.3\% (median $=17.5 \%$ ) depending on the age group. The results of a Saudi modern study predicted an increase of smokers number in the country to 10 million smokers by 2020. The current number of smokers in Saudi Arabia is approximately 6 million, and they spend around 21 billion Saudi Riyal on smoking annually. Clearly smoking is a prevalent problem among Saudis that requires intervention for eradication. Persistent education of the health hazards related to smoking is recommended particularly at early ages in order to prevent initiation of smoking $[1,2]$. Tobacco use is considered a disease that can spread through social contact in a way very similar to the spread of infectious diseases.

Like many infectious diseases, mathematical models can be used to understand the spread of smoking and to predict the impact of smokers on the community in order to help reducing the number of smokers. Castillo-Garsow et al. [3] presented a general epidemiological model to describe the dynamics of Tobacco use and they considered the effect of peer presure, relapse, counselling and treetment. In their model the population was devided into nonsmokers, smokers and smokers who quit smoking. Later, this mathematical model was refined by Sharomi and Gumel [4], they introduced a new class $Q_{t}$ of smokers who temporarily quit smoking. They concluded that the smokingfree equilibrium is globally-asymptotically stable whenever a certain threshold, known as the smokers-generation number, is less than unity, and unstable if this threshold is greater than unity. The public health implication of this result is that the number of smokers in the community will be effectively controlled (or eliminated) at equilibrium 
point if the threshold is made to be less than unity. Such a control is not feasible if the threshold exceeds unity. Later, Lahrouz et al. [5] proved the global stability of the unique smoking-present equilibrium state of the mathematical model developed by Sharomi and Gumel. Zaman [6] derived and analyzed a smoking model taking into account the occasional smokers compartment, and later [7] he extended the model to consider the possibility of quitters becoming smokers again. Erturk et al [8] introduced fractional derivatives into the model and studied it numerically. Zeb et al. [9] presented a new giving up smoking model based on the model in [6] for which the interaction term is the square root of potential and occasional smokers. Van Voorn and Kooi [10] presented a three compartment smoking model which was studied using brute force simulations for the short term dynamics and bifurcation analysis for the long-term dynamics. In 2013 [11], we adopted the model developed and studied in [4, 5] and considered the effect of peer pressure on temporarily quitters. By this we mean the effect of smokers on temporarily quitters which is considered one of the main causes of their relapse.

In this paper, we introduce a new model based on the model in [11] by considering the possibility of temporarily quitters becoming permanent quitters and the impact of this transformation on the existence and stability of equilibrium points. The aim of this work, is to analyze the model by using stability theory of non-linear differential equations and supporting the results with numerical simulation. The paper is organized as follows: In section 2 , we explain the formulation of the model. In section 3, the smoking generation number is calculated and the equilibria are found. The stability of the equilibria is investigated in section 4 . In section 5, we present numerical simulations to support our results. Finally, we end the paper with a conclusion and a comparison between our two models in section 6 ..

\section{Formulation os the model}

Let the total population size at time $t$ be denoted by $N(t)$. We divide the population $N(t)$ into four subclasses, potential smokers (non-smoker) $P(t)$, smokers $S(t)$, smokers who temporarily quit smoking $Q_{t}(t)$ and smokers who permanently quit smoking $Q_{p}(t)$ such that $N(t)=P(t)+S(t)+Q_{t}(t)+Q_{p}(t)$.In [11] we studied the dynamics of smoking by the following four non-linear differential equations:

$$
\begin{aligned}
\frac{d P}{d t} & =\mu-\mu P-\beta P S \\
\frac{d S}{d t} & =-(\mu+\gamma) S+\beta P S+\alpha S Q_{t} \\
\frac{d Q_{t}}{d t} & =-(\mu+\alpha S) Q_{t}+\gamma(1-\sigma) S \\
\frac{d Q_{p}}{d t} & =-\mu Q_{p}+\sigma \gamma S .
\end{aligned}
$$

where $\beta$ is the contact rate between potential smokers and smokers, $\mu$ is the rate of natural death, $\alpha$ is the contact rate between smokers and temporary quitters who revert back to smoking, $\gamma$ is the rate of quitting smoking, $(1-\sigma)$ is the fraction of smokers who temporarily quit smoking (at a rate $\gamma$ ), $\sigma$ is the remaining fraction of smokers who permanently quit smoking (at a rate $\gamma$ ). In this paper, we add a new factor to the model: What happens if some of the temporary quitters decide to quit smoking permanently? What impact would this factor have on the model analysis? To answer these questions we consider the following model

$$
\begin{aligned}
\frac{d P}{d t} & =\mu-\mu P-\beta P S \\
\frac{d S}{d t} & =-(\mu+\gamma) S+\beta P S+\alpha S Q_{t}, \\
\frac{d Q_{t}}{d t} & =-(\mu+\alpha S) Q_{t}+\gamma(1-\sigma) S-\varepsilon Q_{t}, \\
\frac{d Q_{p}}{d t} & =-\mu Q_{p}+\sigma \gamma S+\varepsilon Q_{t} .
\end{aligned}
$$


where $\varepsilon$ is the rate at which temporary quitters quit smoking permanently. We assume that the class of potential smokers is increased by the recruitment of individuals at a rate $\mu$. In model 2 , the total population is constant and $P(t), S(t), Q_{t}(t), Q_{p}(t)$ are respectively the proportions of potential smokers, smokers, temporarily quitters and permanent quitters where $P(t)+S(t)+Q_{t}(t)+Q_{p}(t)=1$. Instead of analyzing model 2 we will only consider the following subsystem:

$$
\begin{aligned}
\frac{d P}{d t} & =\mu-\mu P-\beta P S, \\
\frac{d S}{d t} & =-(\mu+\gamma) S+\beta P S+\alpha S Q_{t}, \\
\frac{d Q_{t}}{d t} & =-\mu Q_{t}-\alpha S Q_{t}+\gamma(1-\sigma) S-\varepsilon Q_{t} .
\end{aligned}
$$

Clearly, $\frac{d P}{d t}+\frac{d S}{d t}+\frac{d Q_{t}}{d t} \leqslant \mu-\mu\left(P+S+Q_{t}\right)$. Therefore, as in [11] it can be shown that $\lim _{t \longrightarrow \infty} \sup N_{1}(t) \leqslant 1$. Thus, the considered region for model 3 is:

$$
\Gamma=\left\{\left(P, S, Q_{t}\right): P+S+Q_{t} \leqslant 1, P>0, S \geqslant 0, Q_{t} \geqslant 0\right\}
$$

$\Gamma$ is positively invariant. i.e. every solution of model 3 , with initial conditions in $\Gamma$ remains there for all $t>0$.

\section{Smoking generation number and equilibria}

Model 3 have two equilibria : the smoking-free equilibrium $E_{o}=(1,0,0)$ and the smoking-present equilibrium $E^{*}=\left(P^{*}, S^{*}, Q_{t}^{*}\right)$, where

$$
\begin{aligned}
P^{*} & =\frac{\mu}{\mu+\beta S^{*}}, \\
Q_{t}^{*} & =\frac{\gamma(1-\sigma) S^{*}}{\mu+\varepsilon+\alpha S^{*}},
\end{aligned}
$$

and $S^{*}$ satisfies the equation

$$
S^{* 2}+\left(\frac{\mu((\mu+\varepsilon) \beta+\gamma \beta+\alpha(\mu-\beta)+\alpha \gamma \sigma)+\gamma \beta \varepsilon}{\beta \alpha(\mu+\gamma \sigma)}\right) S^{*}+\frac{\mu(\mu+\varepsilon)(\mu+\gamma-\beta)}{\beta \alpha(\mu+\gamma \sigma)}=0 .
$$

We will find the smoking generation number $R_{0}$ by the method of next generation matrix [12, 13]. Let $X=$ $\left(S, Q_{t}, P\right)$, then model 3 can be rewritten as: $X^{\prime}=\mathfrak{F}(X)-\mathfrak{V}(X)$ such that

$$
\mathfrak{F}(X)=\left[\begin{array}{c}
\beta P S \\
0 \\
0
\end{array}\right], \mathfrak{V}(X)=\left[\begin{array}{c}
(\mu+\gamma) S-\alpha S Q_{t} \\
(\mu+\alpha S) Q_{t}-\gamma(1-\sigma) S-\varepsilon Q_{t} \\
-\mu+\mu P+\beta P S
\end{array}\right] .
$$

By calculating the Jacobian matrices at $E_{o}$, we find that $D\left(\mathfrak{F}\left(E_{o}\right)\right)=\left[\begin{array}{cc}F & 0 \\ 0 & 0\end{array}\right]$ and $D\left(\mathfrak{V}\left(E_{o}\right)\right)=\left[\begin{array}{cc}V & 0 \\ J_{1} & J_{2}\end{array}\right]$ where $F=\left[\begin{array}{ll}\beta & 0 \\ 0 & 0\end{array}\right]$ and $V=\left[\begin{array}{cc}(\mu+\gamma) & 0 \\ -\gamma(1-\sigma) & \mu-\varepsilon\end{array}\right]$.

Thus, the next generation matrix is $F V^{-1}=\left[\begin{array}{cc}\beta /(\mu+\gamma) & 0 \\ 0 & 0\end{array}\right]$ and the smoking generation number $R_{0}$ is the spectral radius $\rho\left(F V^{-1}\right)=\beta /(\mu+\gamma)$.

The positive smoking-present equilibrium $E^{*}=\left(P^{*}, S^{*}, Q_{t}^{*}\right)$ where $S^{*}$ is a solution to equation 4 and so it can be written as $S_{1,2}^{*}=\frac{1}{2}\left(-A_{1} \pm \sqrt{A_{1}^{2}-4 A_{2}}\right)$, where $A_{1}$ and $A_{2}$ are given by

$$
\begin{aligned}
& A_{1}=\frac{\mu((\mu+\varepsilon) \beta+\gamma \beta+\alpha(\mu-\beta)+\alpha \gamma \sigma)+\gamma \beta \varepsilon}{\beta \alpha(\mu+\gamma \sigma)}, \\
& A_{2}=\frac{\mu(\mu+\varepsilon)(\mu+\gamma-\beta)}{\beta \alpha(\mu+\gamma \sigma)}=\frac{\mu(\mu+\varepsilon)(\mu+\gamma)\left(1-R_{0}\right)}{\beta \alpha(\mu+\gamma \sigma)}
\end{aligned}
$$

If $R_{0}>1$,then $A_{2}<0$ and $\sqrt{A_{1}^{2}-4 A_{2}}>A_{1}$. So we have only one positive solution $S^{*}=\frac{1}{2}\left(-A_{1}+\sqrt{A_{1}^{2}-4 A_{2}}\right)$. 
If $R_{0}=1$ and $\mu \geqslant \beta$, then $A_{2}=0$ and there are no positive solutions. If $R_{0}<1$ and $\mu \geqslant \beta$, then $A_{1}>0$ and $A_{2}>0$, and there are no positive solutions. These results are summarized below.

(i) If $R_{0} \leqslant 1$ and $\mu \geqslant \beta$, then there is no positive equilibrium point,

(ii) If $R_{0}>1$, then we have one positive equilibrium point $E^{*}$.

\section{Stability analysis}

\subsection{Local stability}

First, we give our first result about the local stability of $E_{0}$ :

Theorem 1 If $R_{0}<1$, the smoking-free equilibrium point $E_{0}$ is locally asymptotically stable. If $R_{0}=1$, E $E_{0}$ is locally stable. If $R_{0}>1, E_{0}$ is unstable.

Proof. Evaluating the Jacobian matrix of model 3 at $E_{0}$ gives

$$
J\left(E_{0}\right)=\left[\begin{array}{ccc}
-\mu & -\beta & 0 \\
0 & -(\mu+\gamma)+\beta & 0 \\
0 & \gamma(1-\sigma) & -\mu-\varepsilon
\end{array}\right] \text {. }
$$

Thus, the eigenvalues of $J\left(E_{0}\right)$ are $\lambda_{1}=-\mu, \lambda_{2}=-\mu-\varepsilon<0, \lambda_{3}=-(\mu+\gamma)+\beta$. Clearly $\lambda_{3}$ is negative if $R_{0}<1$. So all eigenvalues are negative if $R_{0}<1$, and hence, $E_{0}$ is locally asymptotically stable. If $R_{0}=1$, then $\lambda_{3}=0$ and $E_{0}$ is locally stable. If $R_{0}>1$, then $\lambda_{3}>0$ which means that there exist a positive eigenvalue. So, $E_{0}$ is unstable.

Next, we investigate the local stability of the positive equilibrium $E^{*}$ by using the following lemma.

Lemma $2[15,16,17]$. Let $M$ be a $3 \times 3$ real matrix. If $\operatorname{tr}(M)$, $\operatorname{det}(M)$ and $\operatorname{det}\left(M{ }^{[2]}\right)$ are all negative, then all of the eigenvalues of $M$ have negative real part.

$M^{[2]}$ in the previous lemma is known as the second additive compound matrix with the following definition.

Definition 1 [18] (Second additive compound matrix). Let $A=\left(a_{i j}\right)$ be an $n \times n$ real matrix. The second additive compound of $A$ is the matrix $A^{[2]}=\left(b_{i j}\right)$ defined as follows:

$$
\begin{aligned}
& n=2: A^{[2]}=a_{11}+a_{22} \\
& n=3: A^{[2]}=\left|\begin{array}{ccc}
a_{11}+a_{22} & a_{23} & -a_{13} \\
a_{32} & a_{11}+a_{33} & a_{12} \\
-a_{31} & a_{21} & a_{22}+a_{33}
\end{array}\right|
\end{aligned}
$$

Theorem 3 The smoking-present equilibrium $E^{*}$ is locally asymptotically stable if $\alpha \leqslant \beta$.

Proof. Linearizing model 3 at the equilibrium $E^{*}=\left(P^{*}, S^{*}, Q_{t}^{*}\right)$ gives

$$
J\left(E^{*}\right)=\left[\begin{array}{ccc}
-\mu-\beta S^{*} & -\beta P^{*} & 0 \\
\beta S^{*} & 0 & \alpha S^{*} \\
0 & -\alpha Q_{t}^{*}+\gamma(1-\sigma) & -(\mu+\varepsilon)-\alpha S^{*}
\end{array}\right]
$$

The second additive compound matrix $J^{[2]}\left(E^{*}\right)$ is

$$
J^{[2]}\left(E^{*}\right)=\left[\begin{array}{ccc}
-\mu-\beta S^{*} & \alpha S^{*} & 0 \\
-\alpha Q_{t}^{*}+\gamma(1-\sigma) & -2 \mu-\varepsilon-\beta S^{*}-\alpha S^{*} & -\beta P^{*} \\
0 & \beta S^{*} & -\mu-\varepsilon-\alpha S^{*}
\end{array}\right]
$$

with the following trace and determinant,

$\operatorname{tr}\left(J\left(E^{*}\right)\right)=-2 \mu-\varepsilon-\beta S^{*}-\alpha S^{*}<0$, 


$$
\begin{aligned}
\operatorname{det}\left(J\left(E^{*}\right)=\right. & \beta \alpha S^{*}\left(\mu Q_{t}^{*}-\beta P^{*} S^{*}\right)+\mu\left(\alpha \mu Q_{t}^{*}-\beta^{2} P^{*} S^{*}\right)+\varepsilon\left(\alpha \mu Q_{t}^{*}+\beta(\mu+\gamma) S^{*}-2 \beta^{2} P^{*} S^{*}\right) \\
\leqslant & \left.\beta \alpha S^{*}\left(\mu Q_{t}^{*}-\beta P^{*} S^{*}\right)+\mu \beta_{t}^{*}-\beta P^{*} S^{*}\right)+\varepsilon\left(2 \beta \mu Q_{t}^{*}+2 \beta \mu S^{*}-2 \beta^{2} P^{*} S^{*}\right) \text { if } \alpha \leqslant \beta \\
& \quad \text { and } \gamma \leqslant \mu \\
= & \mu\left(\beta \alpha S^{*}+\mu \beta\right)\left(Q_{t}^{*}+P^{*}-1\right)+2 \varepsilon \beta \mu\left(Q_{t}^{*}+S^{*}+P^{*}-1\right)<0 \\
& \quad \text { since } Q_{t}^{*}+S^{*}+P^{*}<1
\end{aligned}
$$

and

$$
\begin{aligned}
\operatorname{det}\left(J^{[2]}\left(E^{*}\right)\right)= & -\left(\mu+\beta S^{*}\right)\left[\left(2 \mu+\varepsilon+\beta S^{*}+\alpha S^{*}\right)\left(\mu+\varepsilon+\alpha S^{*}\right)+\beta^{2} P^{*} S^{*}\right] \\
& +\alpha S^{*}\left[\left(-\alpha Q_{t}^{*}+\gamma(1-\sigma)\right)\left(\mu+\varepsilon+\alpha S^{*}\right)\right] \\
= & -\left(\mu+\beta S^{*}\right)\left[2 \mu(\mu+\varepsilon)+\varepsilon(\mu+\varepsilon)+\beta S^{*}(\mu+\varepsilon)+\mu \alpha S^{*}+\varepsilon \alpha S^{*}+\right. \\
& \left.\left.\beta \alpha S^{*^{2}}+\alpha^{2} S^{*^{2}}+\beta^{2} P^{*} S^{*}\right)\right]-\alpha(\mu+\varepsilon)(\mu-\gamma) S^{*}-\mu^{2} \alpha S^{*}-\alpha \beta S^{*^{2}} \\
& (\mu+\varepsilon)-\alpha(\mu \beta-\alpha \gamma) S^{*^{2}}-\alpha^{2} S^{*} Q_{t}^{*}\left(\mu+\varepsilon+\alpha S^{*}\right)-\alpha \gamma \sigma S^{*}\left(\mu+\varepsilon+\alpha S^{*}\right) \\
< & 0 \text { if } \alpha \leqslant \beta \text { and } \gamma \leqslant \mu .
\end{aligned}
$$

Hence, $E^{*}$ is locally asymptotically stable if $\alpha \leqslant \beta$ and $\gamma \leqslant \mu$.

\subsection{Global stability}

We will prove the global stability of $E_{0}$ when $\beta \leqslant \mu$.

Theorem 4 (Global stability of $E_{0}$ ) If $\beta \leqslant \mu$, then $E_{0}$ is globally asymptotically stable in $\Gamma$. (Note that $\beta \leqslant \mu$ implies that $\left.R_{0}<1\right)$.

Proof. First, it should be noted that $P<1$ in $\Gamma$ for all $t>0$. Consider the following Liapunov function [19]

$$
\begin{aligned}
L & =S+Q_{t}, \\
\frac{d L}{d t} & =-(\mu+\gamma) S+\beta P S+\alpha S Q_{t}-(\mu+\varepsilon) Q_{t}-\alpha S Q_{t}+\gamma(1-\sigma) S \\
& \leqslant(-\mu+\beta) S-(\mu+\varepsilon) Q_{t} \leqslant 0 .
\end{aligned}
$$

$\frac{d L}{d t}<0$ for $\beta \leqslant \mu$, and $\frac{d L}{d t}=0$ only if $S=0$ and $Q_{t}=0$. Therefore, the only trajectory of the system on which $\frac{d L}{d t}=0$ is $E_{0}$. Hence, by Lasalle's invariance principle, $E_{0}$ is globally asymptotically stable in $\Gamma$.

To prove the global stability of $E^{*}$ we need the following theorem $[15,20]$

Theorem 5 Let $g\left(P, S, Q_{t}\right)=\left\{g_{1}\left(P, S, Q_{t}\right), g_{2}\left(P, S, Q_{t}\right), g_{3}\left(P, S, Q_{t}\right)\right\}$ be a vector field which is piecewise smooth on $\Gamma^{*}$, and which satisfies the conditions: $g . f=0,(\operatorname{curl} g) \cdot \vec{n}<0$ in the interior of $\Gamma^{*}$, where $\vec{n}$ is the normal vector to $\Gamma^{*}$ and $f=\left(f_{1}, f_{2}, f_{3}\right)$ is a lipschitz continuous field in the interior of $\Gamma^{*}$ and curl $g=\left(\frac{\partial g_{3}}{\partial S}-\frac{\partial g_{2}}{\partial Q_{t}}\right) \vec{i}-$ $\left(\frac{\partial g_{3}}{\partial P}-\frac{\partial g_{1}}{\partial Q_{t}}\right) \vec{j}+\left(\frac{\partial g_{2}}{\partial P}-\frac{\partial g_{1}}{\partial S}\right) \vec{k}$. Then the differential equation system $P=f_{1}, S=f_{2}, Q_{t}=f_{3}$ has no periodic solutions, homoclinic loops and oriented phase polygons inside $\Gamma^{*}$.

Now, let $\Gamma^{*}=\left\{\left(P, S, Q_{t}\right): P+\left(\frac{\mu+\gamma \sigma}{\mu}\right) S+\left(\frac{\mu+\varepsilon}{\mu}\right) Q_{t}=1, P>0, S \geqslant 0, Q_{t} \geqslant 0\right\}$. Then $\Gamma^{*} \subset \Gamma, \Gamma^{*}$ is positively invariant and $E^{*} \in \Gamma^{*}$.

Theorem 6 model 3 has no periodic solutions, homoclinic loops and oriented phase polygons inside the invariant region $\Gamma^{*}$.

Proof. Let $f_{1}, f_{2}$ and $f_{3}$ denote the right hand side of equations in model 3 , respectively. Using $P+\left(\frac{\mu+\gamma \sigma}{\mu}\right) S+$ $\left(\frac{\mu+\varepsilon}{\mu}\right) Q_{t}=1$ to rewrite $f_{1}, f_{2}$ and $f_{3}$ in the equivalent forms, we have

$$
f_{1}(P, S)=\mu-\mu P-\beta P S,
$$




$$
\begin{aligned}
& f_{1}\left(P, Q_{t}\right)=\mu-\mu P-\beta P\left[\frac{\mu}{\mu+\gamma \sigma}\left(1-P-\left(\frac{\mu+\varepsilon}{\mu}\right) Q_{t}\right)\right], \\
& f_{2}(P, S)=-(\mu+\gamma) S+\beta P S+\alpha S\left[\frac{\mu}{\mu+\varepsilon}\left(1-P-\left(\frac{\mu+\gamma \sigma}{\mu}\right) S\right)\right], \\
& f_{2}\left(S, Q_{t}\right)=-(\mu+\gamma) S+\beta\left[1-\left(\frac{\mu+\varepsilon}{\mu}\right) Q_{t}-\left(\frac{\mu+\gamma \sigma}{\mu}\right) S\right] S+\alpha S Q_{t}, \\
& f_{3}\left(P, Q_{t}\right)=-(\mu+\varepsilon) Q_{t}-\left[\alpha Q_{t}-\gamma(1-\sigma)\right]\left[\frac{\mu}{\mu+\gamma \sigma}\left(1-P-\left(\frac{\mu+\varepsilon}{\mu}\right) Q_{t}\right)\right], \\
& f_{3}\left(S, Q_{t}\right)=-(\mu+\varepsilon) Q_{t}-\alpha S Q_{t}+\gamma(1-\sigma) S .
\end{aligned}
$$

Let $g=\left(g_{1}, g_{2}, g_{3}\right)$ be a vector field such that:

$$
\begin{aligned}
g_{1}= & \frac{f_{3}\left(P, Q_{t}\right)}{P Q_{t}}-\frac{f_{2}(P, S)}{P S} \\
= & -\frac{\varepsilon}{P}-\frac{\alpha \mu}{P(\mu+\gamma \sigma)}+\frac{\alpha \mu}{\mu+\gamma \sigma}+\frac{\alpha(\mu+\varepsilon) Q_{t}}{P(\mu+\gamma \sigma)}+\frac{\mu \gamma(1-\sigma)}{P Q_{t}(\mu+\gamma \sigma)}-\frac{\mu \gamma(1-\sigma)}{Q_{t}(\mu+\gamma \sigma)} \\
& -\frac{(\mu+\varepsilon) \gamma(1-\sigma)}{P(\mu+\gamma \sigma)}+\frac{\gamma}{P}-\beta-\frac{\alpha \mu}{(\mu+\varepsilon) P}+\frac{\alpha \mu}{\mu+\varepsilon}+\alpha\left(\frac{\mu+\gamma \sigma}{\mu+\varepsilon}\right) \frac{S}{P}, \\
g_{2}= & \frac{f_{1}(P, S)}{P S}-\frac{f_{3}\left(S, Q_{t}\right)}{S Q_{t}} \\
= & \frac{\mu}{P S}-\beta+\frac{\varepsilon}{S}+\alpha-\frac{\gamma(1-\sigma)}{Q_{t}}, \\
g_{3}= & \frac{f_{2}\left(S, Q_{t}\right)}{S Q_{t}}-\frac{f_{1}\left(P, Q_{t}\right)}{P Q_{t}} \\
= & -\frac{(\mu+\gamma)}{Q_{t}}+\frac{\beta}{Q_{t}}-\beta\left(\frac{\mu+\varepsilon}{\mu}\right)-\frac{\beta(\mu+\gamma \sigma) S}{\mu Q_{t}}+\alpha-\frac{\mu}{P Q_{t}}+\frac{\mu}{Q_{t}}+\frac{\beta \mu}{Q_{t}(\mu+\gamma \sigma)} \\
& -\frac{\beta \mu P}{Q_{t}(\mu+\gamma \sigma)}-\frac{\beta(\mu+\varepsilon)}{(\mu+\gamma \sigma)} .
\end{aligned}
$$

Since the alternate forms of $f_{1}, f_{2}$ and $f_{3}$ are equivalent in $\Gamma$, then

$$
\begin{aligned}
g . f & =g_{1} f_{1}+g_{2} f_{2}+g_{3} f_{3} \\
& =\frac{f_{3} f_{1}}{P Q_{t}}-\frac{f_{2} f_{1}}{P S}+\frac{f_{1} f_{2}}{P S}-\frac{f_{3} f_{2}}{S Q_{t}}+\frac{f_{2} f_{3}}{S Q_{t}}-\frac{f_{1} f_{3}}{P Q_{t}}=0 .
\end{aligned}
$$

So, $g . f=0$ on $\Gamma^{*}$. Using the normal vector $\vec{n}=\left(1, \frac{\mu+\gamma \sigma}{\mu}, \frac{\mu+\varepsilon}{\mu}\right)$ to $\Gamma^{*}$, we can see that

$$
\begin{aligned}
(\operatorname{curl} g) \cdot \vec{n} & \leq-\frac{\beta \gamma \sigma}{\mu Q_{t}}-\frac{\gamma(1-\sigma)}{P Q_{t}^{2}}-\frac{\mu+\gamma \sigma}{P^{2} Q_{t}}-\frac{\mu}{P^{2} S}-\frac{\varepsilon}{\mu P^{2} S}[\mu-\beta P S]-\frac{\alpha \gamma \sigma}{\mu P} \text { if } \alpha \leq \beta \\
& =-\frac{\beta \gamma \sigma}{\mu Q_{t}}-\frac{\gamma(1-\sigma)}{P Q_{t}^{2}}-\frac{\mu+\gamma \sigma}{P^{2} Q_{t}}-\frac{\mu}{P^{2} S}-\frac{\varepsilon}{P S}-\frac{\alpha \gamma \sigma}{\mu P}<0 .
\end{aligned}
$$

Hence, model 3 has no periodic solutions, homoclinic loops and oriented phase polygons inside the invariant region $\Gamma^{*}$

Now we can prove the main result.

Theorem 7 (Global stability of $E^{*}$ ) If $\alpha \leq \beta$, then the smoking -present equilibrium point $E^{*}$ of model 3 is globally asymptotically stable.

Proof. We know that if $R_{0}>1$ in $\Gamma^{*}$, then $E_{0}$ is unstable. Also $\Gamma^{*}$ is a positively invariant subset of $\Gamma$ and the $\omega$ limit set of each solution of model 3 is a single point in $\Gamma^{*}$ since there is no periodic solutions, homoclinic loops and oriented phase polygons inside $\Gamma^{*}$ if $\alpha \leq \beta$. Therefore $E^{*}$ is globally asymptotically stable if $\alpha \leq \beta$. 


\section{Numerical simulations}

In this section, we illustrate some numerical solutions of model 2 for different values of the parameters to compair it with the qualitative results. We use the following parameters: $\alpha=0.25, \sigma=0.4$ and $\varepsilon=0.23$. For $R_{0}<1$, we use $\beta=0.2, \mu=0.04$ and $\gamma=0.3$, and for $\beta \geqslant \alpha$ and $\mu \geqslant \gamma$, we use $\beta=0.5, \mu=0.12$ and $\gamma=0.1$. We use the following different initial values such that $P+S+Q_{t}+Q_{p}=1$ :

$$
\begin{aligned}
& 1-P(0)=0.80301, S(0)=0.10628, Q_{t}(0)=0.08260, Q_{p}(0)=0.00811 \\
& 2-P(0)=0.75000, S(0)=0.16772, Q_{t}(0)=0.07000, Q_{p}(0)=0.01228 \\
& 3-P(0)=0.70000, S(0)=0.21800, Q_{t}(0)=0.05566, Q_{p}(0)=0.02634 \\
& 4-P(0)=0.63400, S(0)=0.28800, Q_{t}(0)=0.04800, Q_{p}(0)=0.03000
\end{aligned}
$$

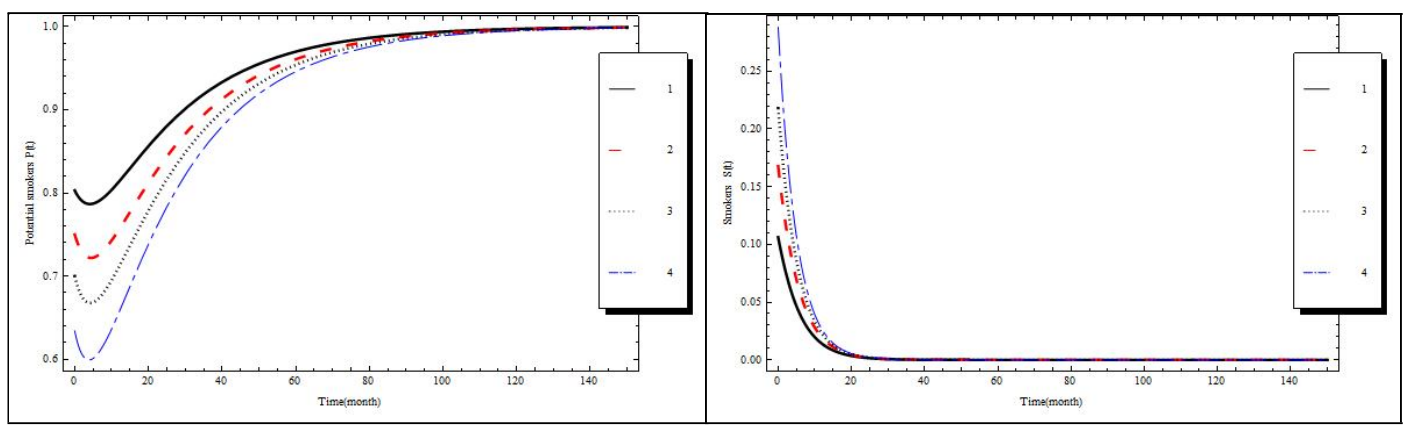

(a)

(b)

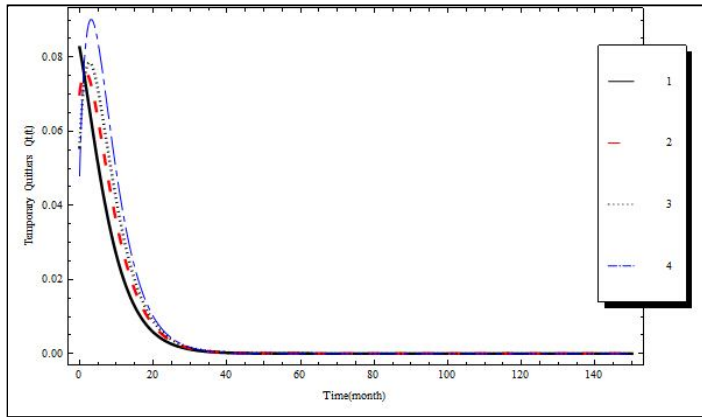

(c)

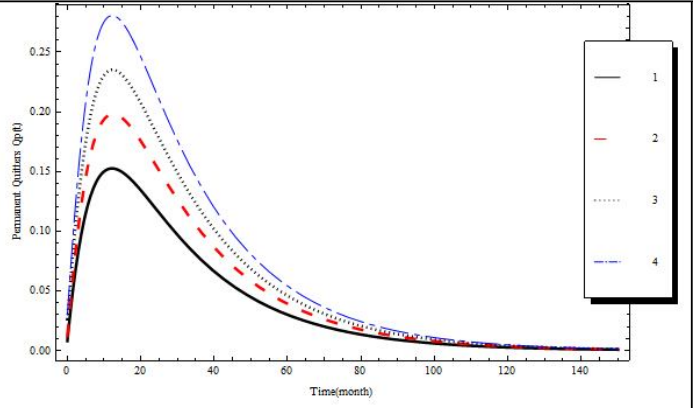

(d)

Fig. 1. Time plots of model 2 with different initial conditions for $R_{0}<1$. (a) Potential smokers; (b) Smokers; (c) Temporary quitters; (d) Permanent quitters.

For $R_{0}=0.588235<1$, Fig. 1(a) shows that the number of potential smokers increases and approaches the total population 1. Fig. 1(b) shows that the number of the smokers decreases and approaches zero . In Fig. 1(c,d), temporary quitters and permanent quitters increase at first, after that they decrease and approach zero. We see from Fig. 1 that for any initial value, the solution curves tend to the equilibrium $E_{0}$, when $R_{0}<1$. Hence, model 2 is locally asymptotically stable about $E_{0}$ for the previous set of parameters.

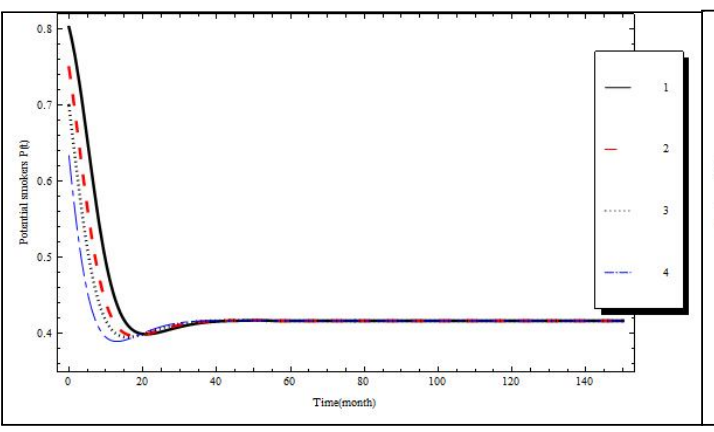

(a)

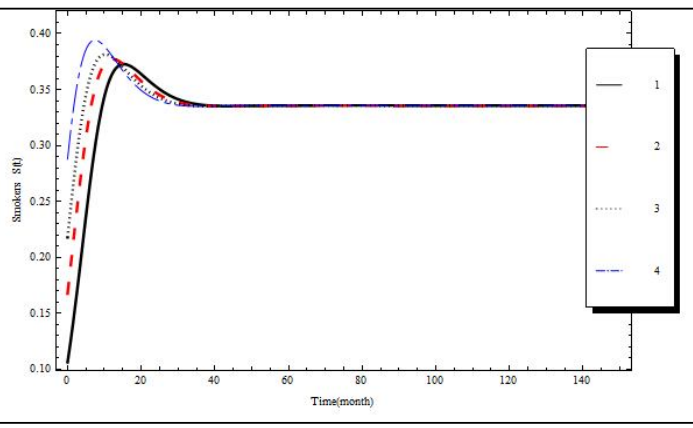

(b) 


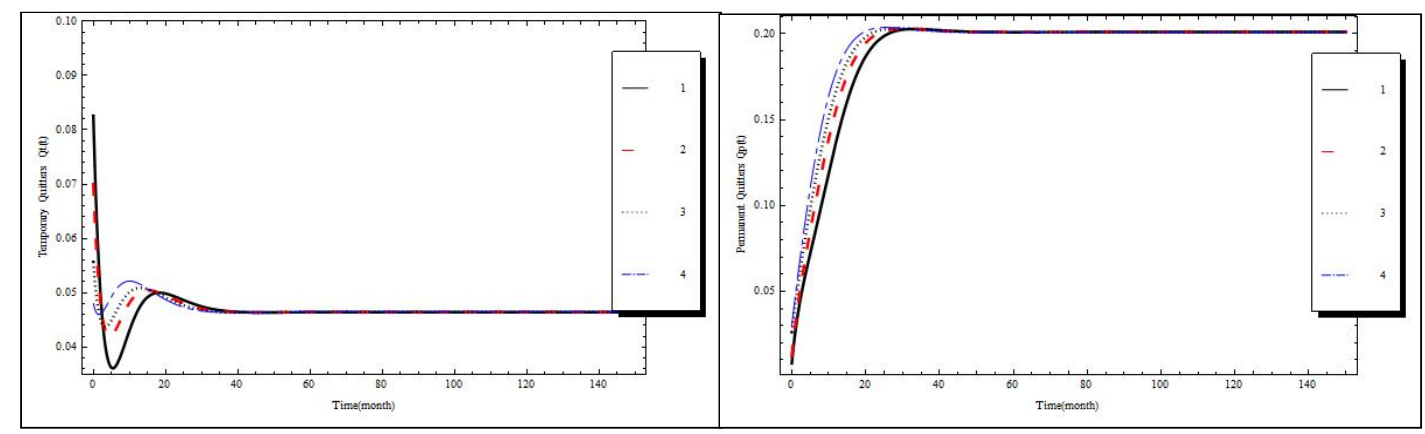

(c)

(d)

Fig. 2. Time plots of model 2 with different initial conditions for $\beta \geqslant \alpha$ and $\mu \geqslant \gamma$. (a) Potential smokers; (b) Smokers; (c) Temporary quitters; (d) Permanent quitters.

In Fig. 2, we use the same parameters and initial values as before with $\beta=0.5, \mu=0.12$ and $\gamma=0.1$. Fig. 2 (a) shows that the number of potential smokers decreases at first, then it approaches $P^{*}$. Fig. 2(b) shows that the number of smokers increases at first, then it decreases slightly and approaches $S^{*}$. In Fig. 2(c), temporary quitters decrease at first, after that they increase and approach $Q_{t}^{*}$. In Fig. 2(d), permanent quitters increase and approach $Q_{p}^{*}$. We see from Fig. 2 that for any initial value, the solution curves tend to the equilibrium $E^{*}$, when $\beta \geqslant \alpha$ and $\mu \geqslant \gamma$. Hence, model 2 is locally asymptotically stable about $E^{*}$ for the above set of parameters..

\section{Discussion and conclusion}

In this paper, we presented a mathematical model to analyze the behavior of a giving up smoking dynamics in a population by considering the possibility of temporarily quitters become permanent quitters. Local asymptotic stability for the smoking-free equilibrium state is obtained when the threshold quantity $R_{0}$ is less than 1 (i.e. when the contact rate $\beta$ between potential smokers and smokers is less than the sum of natural death rate $\mu$ and the quitting rate $\gamma$ ). A Liapunov function is used to show global stability of the smoking-free equilibrium when the contact rate between potential smokers and smokers is less than or equal to the natural death rate $(\beta \leq \mu)$. This means that the number of smokers may be controlled by reducing the contact rate $\beta$ to be less than the natural death rate $\mu$. On the other hand if $\beta \geqslant \alpha$ and $\mu \geqslant \gamma$, then the smoking present equilibrium state is locally asymptotically stable. By showing that this model has no periodic solutions, homoclinic loops and oriented phase polygons inside the invariant region $\Gamma^{*}$, we proved the global asymptotic stability of $E^{*}$ if $\beta \geqslant \alpha$. This means that if $\beta \geqslant \alpha$, smoking will persist. Some numerical simulations are performed to illustrate the findings of the analytical results. For $\beta \geqslant \alpha$, the results show that the smokers population reaches a steady state of approximately $33 \%$ of the total population.

Under the same parameters and initial conditions used in the numerical simulation, we introduce the following figures to compare this model with our previous model in [11].

Near $E_{0}$ : In Fig. 3, we notice that there is no difference in the behavior of solutions of the two models near $E_{0}$, but in Fig. 4, we notice a difference in the behavior of the solutions. The number of temporary quitters increases rapidly at first in model 1 and not in model 2 and we also notice that the number of parmanent quitters increases rapidly at first in model 2 and not in model 1 , but in both models solutions approach $E_{0}$.

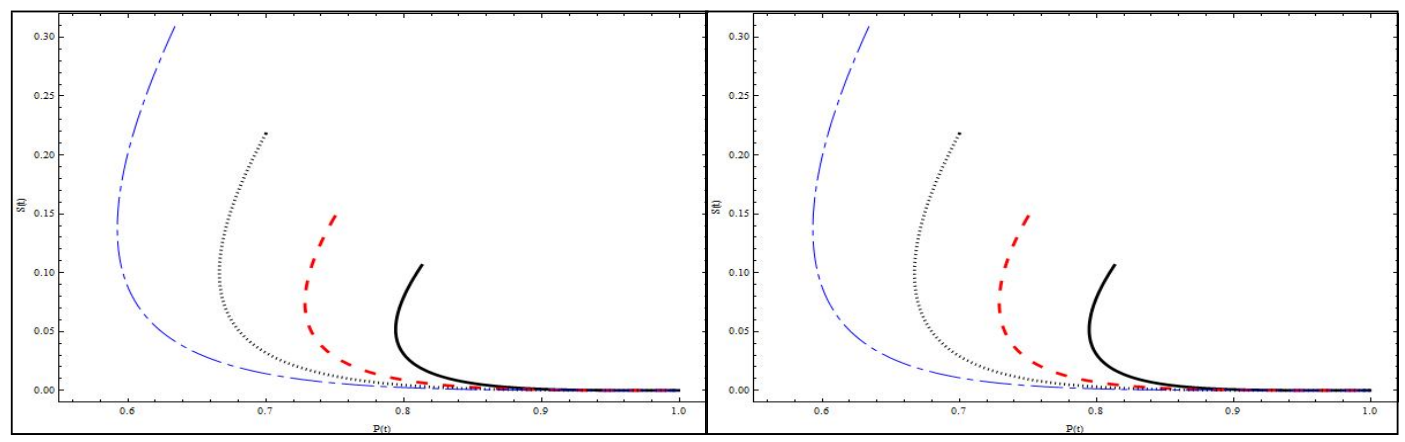

Model ${ }^{1}$ Fig.3 Smokers S vs. potential smokers P Model 2 


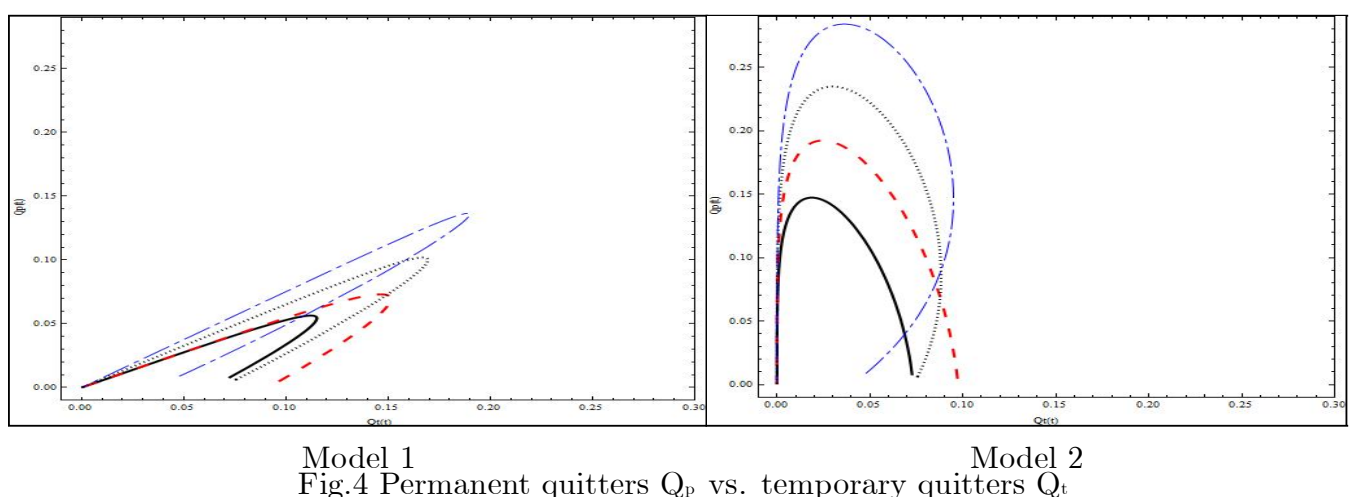

Near $E^{*}$ : we will use the following parameters $\beta=0.5, \mu=0.12, \gamma=0.1, \alpha=0.25, \sigma=0.4$ and $\varepsilon=0.23$ which satisfy local stability conditions for the two models, and use the same initial conditions. In Fig. 5 and Fig. 6 we notice that $S^{*}$ and $Q_{t}^{*}$ are greater in model 1 than in model 2 and $P^{*}$ and $Q_{p}^{*}$ are less in model 1 than in model 2. So, model 2 is better than model 1 in the sense that if smoking persists in the community, the number of smokers is less in model 2.

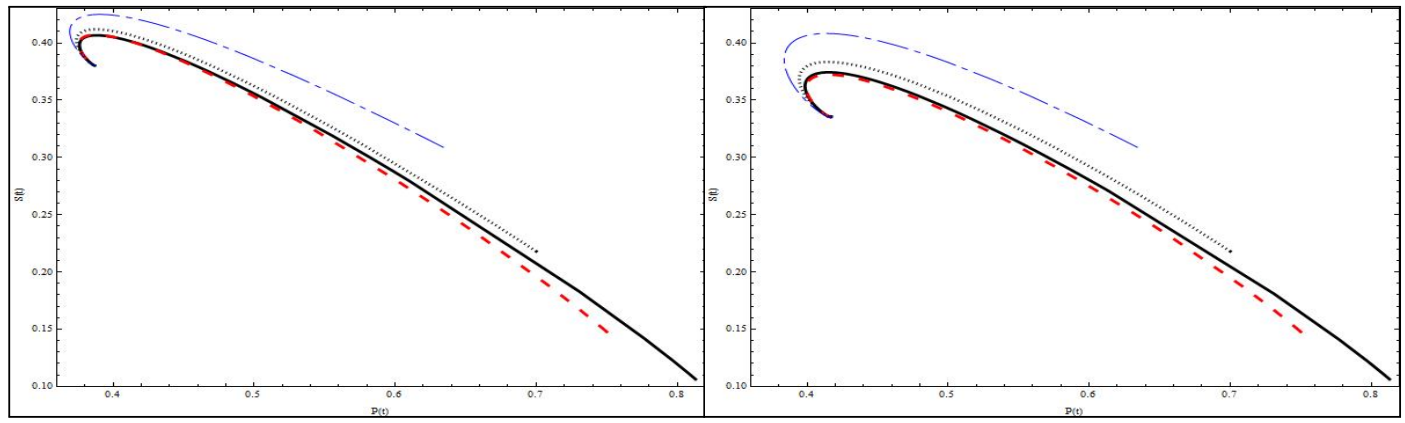

Model ${ }^{1}$ Fig.5 Smokers S vs. potential smokers P

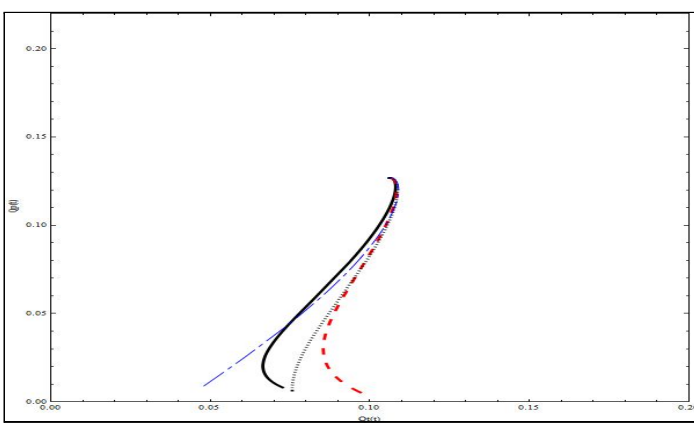

Model 1

\section{References}

[1] MM. Bassiony, Smoking in Saudi Arabia, Saudi Med Journal 30 (2009) 876-881.

[2] World Health Organization report on tobacco, 2013.

http://www.who.int/tobacco/global_report/2013/en/index.html

[3] C. Castillo-Garsow, G. Jordan-Salivia, and A. Rodriguez Herrera, Mathematical models for the dynamics of tobacco use, recovery, and relapse, Technical Report Series BU-1505-M, Cornell University, Ithaca, NY, USA, 1997.

[4] O. Sharomi, A.B. Gumel, Curtailing smoking dynamics: A mathematical modeling approach, Applied Mathematics and Computation 195 (2008) 475-499. 
[5] A. Lahrouz, L. Omari, D. Kiouach, A. Belmaâti, Deterministic and stochastic stability of a mathematical model of smoking, Statistics and Probability Letters 81 (2011) 1276-1284.

[6] G.Zaman, Qualitative Behavior of Giving Up Smoking Models, Bulletin of the Malaysian Mathematical Sciences Society 34 (2011) 403-415.

[7] G.Zaman, Optimal Campaign in the Smoking Dynamics, Computational and Mathematical Methods in Medicine, 2011(2011) Article ID 163834, 9 pages

[8] V.S.Ertürk, G.Zamanb, S.Momanic, A numeric-analytic method for approximating a giving up smoking model containing fractional derivatives, Computers and Mathematics with Applications 64 (2012) 3065-3074

[9] A. Zeb, G. Zaman and S. Momani, Square-root dynamics of a giving up smoking model, Applied Mathematical Modelling 37 (2013) 5326-5334

[10] G.A.K. van Voorn, B.W. Kooi, Smoking epidemic eradication in a eco-epidemiological dynamical model, Ecological Complexity, 14 (2013) 180-189

[11] Z. Alkhudhari, S. Al-Sheikh, S. Al-Tuwairqi, Global Dynamics of a Mathematical Model on Smoking, ISRN Applied Mathematics vol. (2014), Article ID 847075.

[12] P.van den Driessche, J.Watmough, Reproduction numbers and sub-threshhold endemic equilibria for compartmental models of disease transmission, Mathematical Biosciences 180 (2002) 29-48.

[13] F.Brauer, P.van den Driessche and J.Wu, Mathematical Epidemiology, Springer-Verlag (2008).

[14] L.Perko, Differential Equations and Dynamic Systems, Springer Verlag(1991).

[15] L.Cai, X.Li, M.Ghosh, B.Guo, Stability analysis of an HIV/AIDS epidemic model with treatment, Journal of Computational and Applied Mathematics 229 (2009) 313-323.

[16] C. C. McCluskey, P.van den Driessche, Global analysis of two tuberculosis models, Journal of Dynamics and Differential Equation 16 (2004) 139-166.

[17] J.Arino, C.C. McCluskey, and P.van den Driessche, Global results for an epidemic model with vaccination that exhibits backward bifurcation, SIAM, Journal Applied Mathematics 64 (2003) 260-276.

[18] X.Ge, M.Arcak, A sufficient condition for additive D-stability and application to reaction-diffusion models, Systems and Control Letters 58 (2009) 736-74.

[19] M.W.Hirsch, S.Smale, and R.L.Devaney, Differential equations, dynamical systems and an introduction to chaos, Elsevier Academic press (1974).

[20] S.Busenberg, P.van den Driessche, Analysis of a disease transmission model in a population with varying size, Journal of Mathematical Biology 28 (1990) 257-270. 